\title{
Essai d'analyse épistémologique de la théorie synthétique de l'évolution
}

\author{
Michel Delsol, Philippe Sentis, Janine Flatin
}

Face aux critiques formulées actuellement à l'encontre de la "théorie synthétique de l'évolution" et aux questions que certains se posent alors à son sujet, nous avons tenté dans cet article de résumer l'analyse épistémologique de cette théorie que nous avons publiée récemment [1]. Ce beaucoup trop bref exposé veut souligner à la fois la masse et la diversité des observations et expérimentations sur lesquelles est fondée la théorie synthétique et essayer de décrire les différents processus et schémas explicatifs de l'évolution biologique qu'elle propose: du système mutation-sélection bien connu de tous à la construction des organes les plus complexes ou au passage d'un haut taxon à l'autre dans nos phylogenèses (c'est-àdire d'une grande catégorie de la classification à l'autre).

Nous avons insisté, en particulier, sur le jeu dialectique de la sélection directionnelle et de la sélection stabilisante.

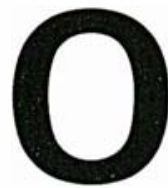
n sait que, pour expliquer le phénomène de l'évolution, il y a eu deux principales théories : celle de l'hérédité des caractères acquis de Lamark et celle des changements héréditaires sélectionnés de Darwin, qui devint, après 1900 , le système des mutations sélectionnées (rappelons que Darwin ne refusait pas le principe de l'hérédité des caractères acquis, mais ne l'acceptera vraiment qu'à la fin de sa vie). A partir de 1908, ce dernier schéma fut pris en charge par la génétique, et notamment par la génétique des populations. Vers la fin des années 1930, il devint la "théorie synthétique de l'évolution". Cette nouvelle synthèse n'est pas, comme on le dit parfois, une théorie de généticiens mais une théorie issue de comparaisons entre les données de la génétique et celles fournies par l'ensemble des sciences de la nature : paléontologie, zoologie, botanique, géographie zoologique, éthologie, biologie moléculaire, etc. Elle est souvent critiquée depuis quelque vingt ans, surtout en raison du rôle qu'elle attribue à la sélection. Pourtant, il suffit de parcourir la table des matières des grandes revues spécialisées : Evolution, American Naturalist, la collection Evolutionary Biology, le Bulletin de la Société zoologique de France, pour voir que les auteurs spécialistes de ces sujets sont, en majorité, mais évidemment avec diverses nuances, synthéticiens. Les critiques sont certainement dues parfois à l'immense complexité de l'actuelle théorie synthétique, que peu d'auteurs ont pris la peine d'étudier suffisamment, mais aussi au fait qu'on lui reproche les interprétations philosophiques de toute nature, du reste souvent contradictoires, que l'on en a tirées : sociobiologie, suivant les cas marxisme, antimarxisme ou athéisme... On oublie que données scientifiques et réflexions philosophiques constituent deux domaines différents du savoir. Nous allons essayer, dans ce texte, de présenter une vue générale de cette théorie. Puisqu'elle est fondée sur un double regard, celui des naturalistes et celui des généticiens, notre exposé devra suivre ce même schéma. Dans un livre récent [1], nous l'avons résumée en 20 propositions* correspondant les unes aux concepts issus du premier regard, les concepts descriptifs, les autres au second regard, c'est-à-dire les concepts explicatifs (figure 1, p. 1080). Nous reprendrons ce plan ici.

\section{Concepts descriptifs}

On peut les résumer en cinq points.

Richesse et diversité du monde vivant

On a reconnu aujourd'hui environ 2 millions d'espèces vivantes, mais on est certainement très loin d'avoir terminé l'inventaire des espèces et sous-

\footnotetext{
* Ces propositions figurent en chiffres romains dans la figure 1 et sont aussi appelées à plusieurs reprises dans le texte.
} 


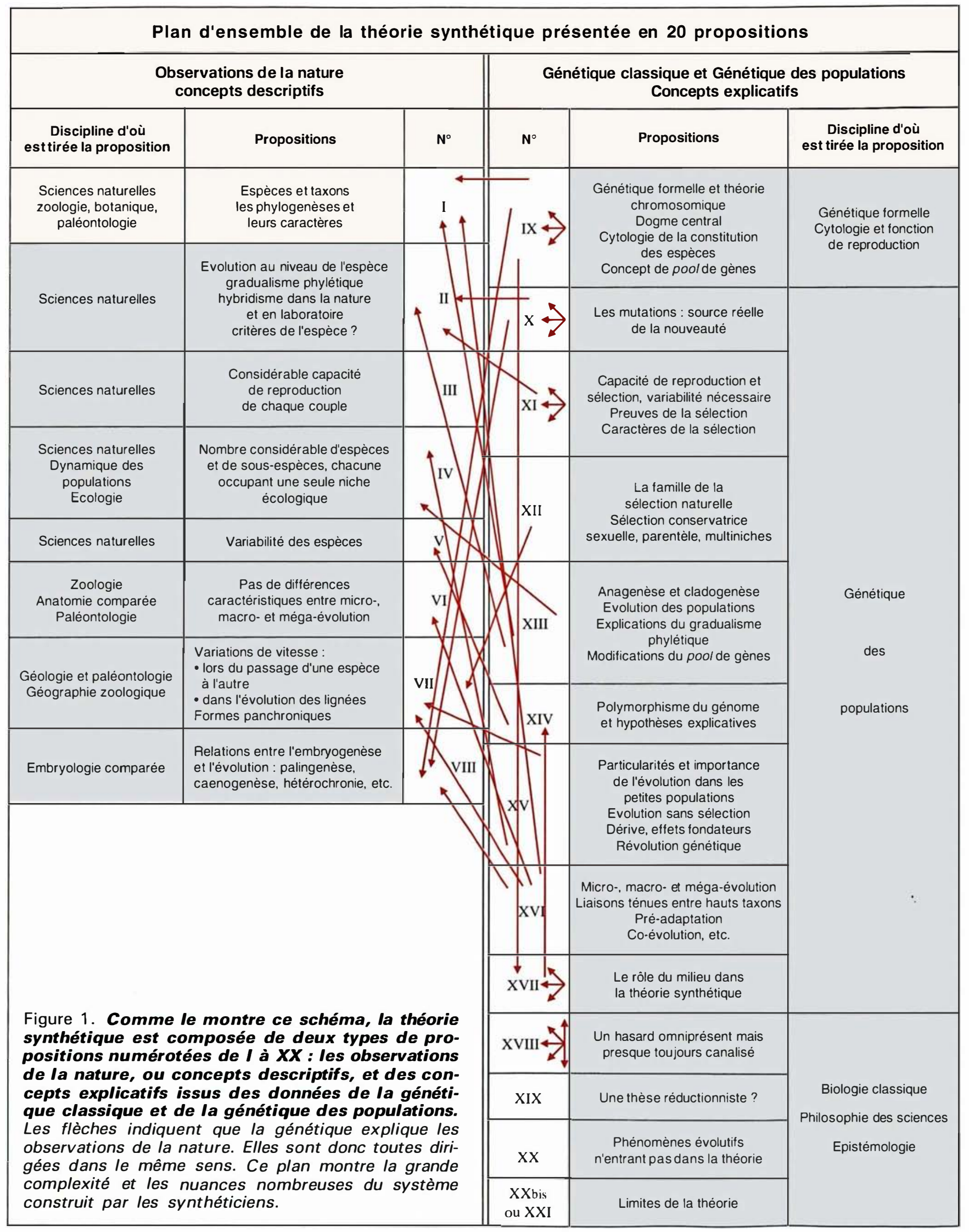


espèces qui peuplent la terre ; il y en aurait, d'après certaines projections, 20 à 30 millions. On décrit par exemple plus de 15000 espèces nouvelles d'insectes chaque année. Darwin avait déjà compris que l'ensemble du monde animal et végétal ressemblait à un immense continuum aux branches multiples. On a défini, à la suite de Simpson, une microévolution: passage d'une sous-espèce à l'autre, une macro-évolution : passage d'une espèce à l'autre, et une méga-évolution : passage d'un haut taxon à l'autre (des reptiles aux mammifères, par exemple). Chaque couple animal ou végétal a, en général, une prolificité considérable et il y a, sauf dans de rares cas (cavernicoles), une mortalité considérable également à chaque génération (propositions I, II, III et IV).

\section{Espèce-polymorphisme-hybridisme}

On admet que, globalement, font partie de la même espèce les populations dont les sujets se croisent entre eux et ne peuvent se croiser qu'entre eux, mais, par obligation pratique, on définit souvent les espèces par des caractères morphologiques. Cependant, on constate, à l'intérieur de chaque espèce, une variabilité considérable, c'est le polymorphisme [2]. En outre, contrairement à ce que l'on a cru pendant longtemps, on découvre de plus en plus chaque jour qu'entre espèces très voisines il y a souvent un hybridisme possible. Dans le genre Drosophila, on connaît actuellement plus de 1500 espèces ; sur 266 essais de croisement entre espèces effectués en laboratoire, dans 170 cas on a obtenu des F1 en abondance, avec au moins un des deux sexes fertile et, dans 40 cas, des hybrides fertiles en tous sens [3]. On pourrait multiplier aujourd'hui ce type d'observation. L'espèce ne paraît donc plus une entité absolue, et on a été amené à penser que la constitution d'une espèce nouvelle peut se réaliser progressivement dans une population [4]. La paléontologie a souvent confirmé ce fait dans les cas où l'on a trouvé assez de fossiles. Jaeger [5], par exemple, a montré que des relations phylogénétiques entre deux espèces considérées comme éloignées en 1935 se sont avérées, en 1962 à la suite de nouvelles fouilles, correspondre probablement à une séquence de formes très voisines. En outre (proposition II), les zoologistes ou botanistes géographes ont compris depuis longtemps que, lorsque deux populations $\mathrm{A} 1$ et $\mathrm{A} 2$ issues d'une même espèce $A$ sont séparées par un isolement géographique, elles évoluent chacune pour leur compte mais en demeurant longtemps très proches : on les dénomme alors espèces vicariantes. On dit aussi qu'elles sont "géminées". Ainsi, on a démontré, il y a longtemps déjà, qu'il existe des centaines d'espèces ou de sous-espèces de poissons, d'échinodermes ou de mollusques géminées de chaque côté de l'isthme de Panama $[6,7]$. En somme, l'espèce n'est pas une entité bien délimitée, mais elle apparaît de plus en plus comme un ensemble extrêmement flou.

Le concept de sous-espèce est, lui aussi, très flou, et il y a à son niveau une valse classificatoire permanente. Chaque espèce ou sous-espèce occupe une niche écologique différente; ce terme ne désigne pas un simple lieu mais tous les caractères de leur habitat (proposition IV) [8].

\section{Méga-évolution}

Ce qui paraît évident pour le passage entre espèces doit être vrai aussi entre les hauts taxa. Les reptiles actuels correspondent à cinq groupes connus de tous : tortues, lézards, crocodiles, serpents, Hatteria. On savait depuis longtemps que c'était chez des reptiles fossiles qu'il fallait rechercher l'origine des mammiferes. On a trouvé 23 espèces fossiles qui constituent une remarquable série d'intermédiaires entre les deux groupes. Un cladogramme donné par Kemp [9, 10] illustre bien ces travaux (figure 2, p. 1082). On est amené à penser, par inférence inductive, qu'il doit en être de même pour les groupes zoologiques ou paléontologiques entre lesquels aujourd'hui nous manquons de fossiles. Avec le temps, ceux-ci devraient être trouvés de même que se sont comblées peu à peu les cases vides de la classification de Mendeleiev.

Une science ancienne mais indispensable, l'anatomie comparée, confirme cette opinion. En suivant la phylogenèse des vertébrés, on peut montrer par exemple que l'on passe dans la nature, par transition insensible, du système cardiaque et vasculaire très simple du poisson au cœur à quatre cavités des vertébrés supérieurs. Un auteur récent (Denton, [11]) a cru pouvoir faire renaître une anatomie comparée typologique : son argumentation est fondée, à ce sujet, sur des manuels élémentaires d'anatomie [12].

\section{Système répétitif $\mathrm{du}$ monde vivant}

Toutes les branches de la biologie nous amènent à penser que le monde vivant s'est construit très souvent par répétition d'un matériel de base : les molécules de la vie ne seraient que 500. Tout est cellulaire et, dans toutes les cellules, il y a un noyau, un appareil de Golgi et "trois classes moyennes de fibres" : les microfilaments d'actine, les filaments intermédiaires et les microtubules [13]. L'anatomie révèle que la majorité des espèces est d'origine métamérique et que, dans le métamère, il y a des unités répétitives: lobules du foie, îlots du pancréas, systèmes de Havers, unités de la rétine, etc. Tout cela suggère une évolution graduelle par addition possible d'unités nouvelles, répétant celles existant déjà. Ce type de construction réduit les apports nécessaires de nouveautés réelles (proposition VI).

\section{Vitesse d'évolution}

En tenant compte de l'histoire phylogénétique reconstruite grâce aux travaux des paléontologistes, des zoologistes et des botanistes, on peut montrer que la vitesse de l'évolution des groupes ou des organes est très variable. Un (xil complexe existe chez les premiers 'vertébrés et ensuite change peu. En revanche, certaines régions de l'oreille se constituent très vite, lors du passage des reptiles aux mammifères. Les formes dites fossiles vivants ne constituent pas des êtres doués de structures d'exception, mais correspondent seulement, dans la courbe de durée de vie des groupes, à ceux qui ont vécu le plus longtemps (proposition VII).

\section{Les concepts explicatifs}

La génétique classique et la génétique des populations expliquent les phéno- 
mènes que nous venons de résumer par le système que l'on désigne abusivement en deux mots : mutationsélection, et qui correspond en réalité à une théorie très complexe. On peut essayer de la schématiser ainsi (figure 1).

\section{Les mutations}

Facteurs essentiels de l'évolution, elles sont beaucoup plus nombreuses qu'on ne le croit habituellement. On a pu estimer leur taux par méiose à une pour 100000 gènes. Le génome humain contient certainement plus de 100000 gènes actifs, chaque homme naît très probablement porteur d'au moins une mutation nouvelle. Elles se réalisent "par hasard "; ce concept est généralement mal compris. Les synthéticiens lui ont donné le sens classique de Cournot [14] : rencontre de séries causales indépendantes. Donc ce n'est pas un phénomène sans cause, ni un phénomène qui peut entraîner n'importe quoi. Le hasard utilise les éléments dont il dispose et les modifie, en l'occurrence ici, un génome. Il ne pourra donc pas effectuer des modifications trop importantes. Une souris ne pourra donner qu'une autre souris de souche légèrement différente mais jamais - hormis exceptions rares - un animal très différent. Les transformations évolutives seront donc lentes; cela s'insère parfaitement dans les concepts descriptifs.

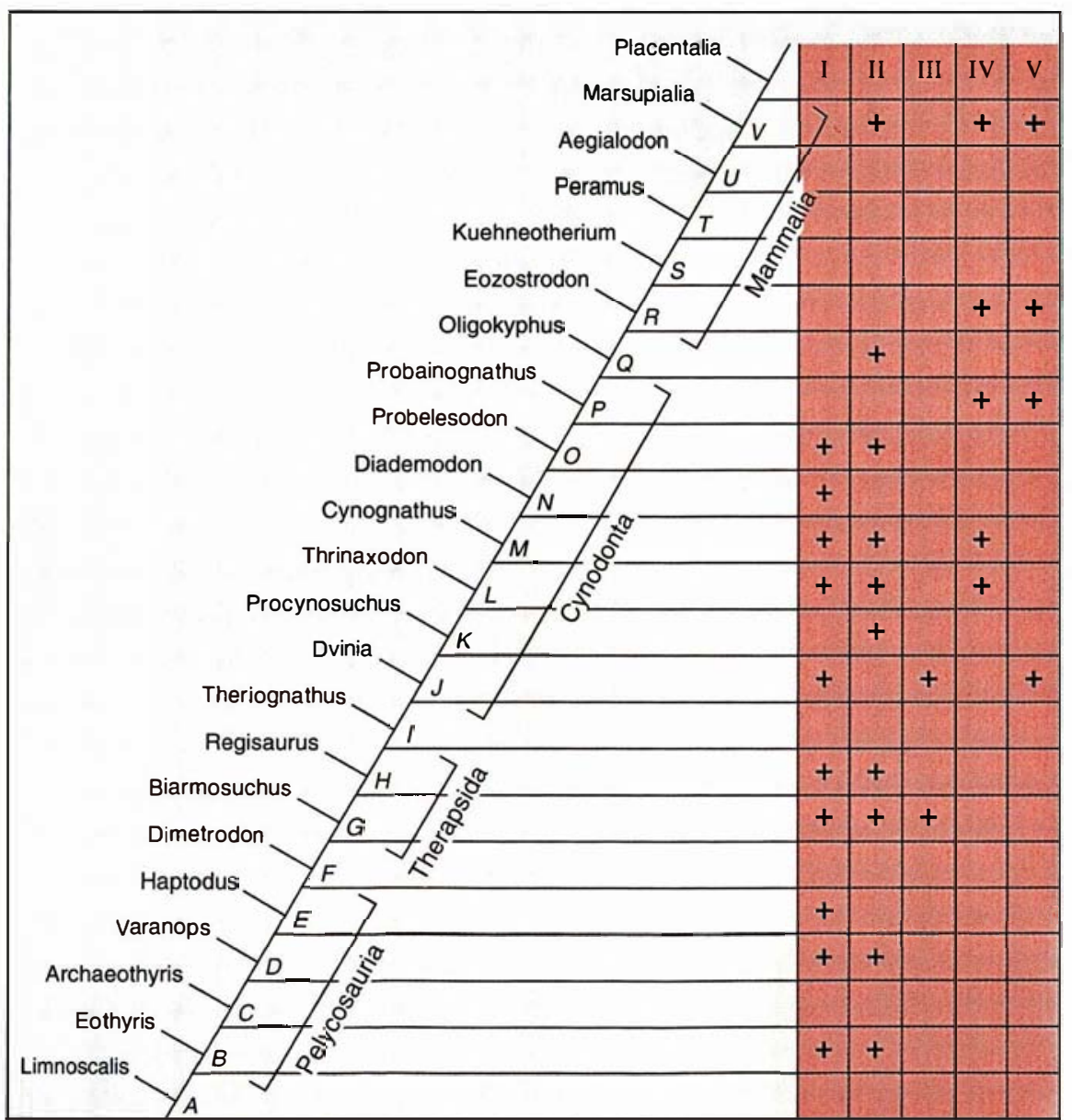

Figure 2. Le passage des reptiles aux mammifères est aujourd'hui suivi, espèce par espèce, par les paléontologistes. Ce cladogramme reproduit un dessin de Kemp en le complétant avec des données de cet auteur lui-même pour montrer les étapes de l'évolution de divers appareils représentatifs des principales différences entre les pièces squelettiques de ces deux groupes. I: marques squelettiques des muscles adducteurs de la joue; II: complexité de la dentition; III : réception des sons et stapes ; $N$ : conduction des sons ; $V$ :

Sélection directionnelle et sélection stabilisante

Darwin, ayant lu Malthus, avait compris la prolificité des espèces. Ayant compris aussi que chaque sujet de chaque espèce présentait de petites variations héréditaires, il admit que la mort ne frappait pas au hasard, mais que souvent survivait le plus apte, nous préférons dire le moins inapte. Cette proposition n'avait pas d'autre argument que la simple logique. En sciences, la simple logique ne suffit pas pour convaincre. Entre 1908 et la fin des années 1930, après la naissance de la génétique moderne, il fut montré par un calcul mathématique que, dans une population panmixique (c'est-àdire une population où tous les individus peuvent se croiser entre eux en théorie, au hasard des rencontres), s'il n'y avait pas de sélection, le pool de gènes de la population ne devait pas changer. Ensuite, il fut établi encore mathématiquement que, dans cette même population, s'il y avait sélection, on pouvait en mesurer les taux. Appuyé sur une démonstration mathématique, le raisonnement darwinien commença à convaincre, mais il lui manquait des preuves expérimentales ou des observations. A cette époque, en France, Teissier et L'Héritier eurent l'idée de placer des drosophiles dans des cages dites "à population " où dans un espace déterminé, avec une quantité de nourriture donnée, ne pouvaient vivre que 3000 mouches. Comme chaque mouche pond 100 œufs par génération, en plaçant dans la cage deux populations ne différant en théorie que par une mutation connue, on devait pouvoir suivre leur développement et voir si l'une était sélectionnée ou bien si la mort frappait au hasard [15]. Depuis cette époque, de très lourdes expérimentations ont démontré que toujours, dans ces cas, un même type de sujet était éliminé ou ramené dans la cage à un certain taux, ce qui suggérait, dans ce dernier exemple, un avantage pour les hétérozygotes. Dans certaines expériences, il fut établi clairement que le remplacement d'un type de population par l'autre était dû à la mortalité des larves ou des adultes, et non à la plus grande fécondité de l'une des deux formes présentes dans la cage [16]. C'était $\mathrm{m} / \mathrm{s} n^{\circ} 10$ vol. 8, décembre 92 
le triomphe "expérimental" de la thèse de la sélection.

En face de toute théorie cependant, un biologiste ne sera parfaitement convaincu que s'il "voit " dans la nature des faits qui démontrent sa réalité. A partir de 1953, ce fut réalisé. Des observations également nombreuses et très précises démontrèrent que la mort ne frappait pas au hasard mais, par exemple, tuait, suivant les circonstances, un certain type de papillon, gris ou noir. Un livre entier a pu être écrit par Ford [17] sur les expériences réalisées dans ce domaine. Cependant, dans les situations ainsi décrites, la sélection provoque le développement d'un sujet possédant un caractère nouveau : on dit qu'elle est directionnelle (prop. $\mathrm{XI}$ ).

Dans d'autres cas au contraire, la sélection élimine la nouveauté parce que celle-ci n'est pas adaptée au milieu et, dans une population donnée, elle aura tendance à maintenir le type moyen. Ce fait a été plus difficile à démontrer que le précédent, car il est plus facile de prouver l'action d'un phénomène provoquant la naissance d'une nouveauté que de démontrer un phénomène maintenant un type moyen. Cependant, Max Hecht [18], par exemple, a bien démontré que, chez le lézard Aristelliger, les plus grandes chances de survie face au hibou appartiennent au type moyen. On connaît un certain nombre d'exemples de ce genre qui démontrent la réalité de ce qu'on appelle la sélection stabilisante [19]. On peut donc considérer que la constitution et le développement des nouveaútés sera le produit d'opportunités dues aux circonstances et de l'équilibre dialectique entre les sélections directionnelle et stabilisante. C'est à cause de cela qu'il existe en même temps sur la terre des bactéries, des fourmis et des hommes et que l'apparition d'un groupe nouveau, à partir d'une population d'un autre groupe, n'empêche pas cette dernière de survivre dans une autre niche écologique.

Le couple mutation-sélectionanagenèse et cladogenèse

- Le ponctualisme. Une expérience typique doit être rappelée : dans des cages à population, on accroît le taux normal de mutations par irradiation aux rayons $\mathrm{X}$. La majorité des mutations étant délétères, on observe une mortalité très importante. Cependant, on constate qu'il apparaît, parmi les mouches irradiées, des sujets mutés qui savent mieux exploiter le milieu que les précédents, et qui remplissent à nouveau les cages. En 1977, Dobzhansky a écrit : "La conclusion inévitable est que, bien que l'effet moyen de nouvelles mutations induites fût délétère, une minorité d'entre elles se sont prouvées favorables en ce sens qu'elles ont permis aux populations d'exploiter avec plus d'efficacité leur environnement sévèrement limité " [20].

Cependant, il est logique de penser que, pour que dans la nature de telles micro-évolutions aboutissent à long terme à des espèces nouvelles ou à des taxa nouveaux, il sera nécessaire que de tels phénomènes se réalisent à l'état naturel avec des mutations en quantité normale dans une population panmictique, mais pendant de longues périodes - milliers, centaines de milliers, voire millions d'années. En somme on est amené à penser que si de telles populations présentent, longtemps, une mutation sélectionnée, plus une mutation sélectionnée, etc., on aura des structures totalement nouvelles. En théorie, on devrait pouvoir alors suivre de telles évolutions en paléontologie. Cela a quelquefois été vu au cours de ces dernières décennies. Dommergues [21] a décrit dans le Lias moyen, au Portugal, une série d'ammonites. Sur trois millions d'années, il a pu estimer que se suivaient plusieurs espèces divisées en deux genres. Gingerich [22] a décrit des séries analogues chez des mammifères. Ces modifications lentes de populations ont été nommées anagenèses (figure 3, p. 1084, parties I et II). Les espèces ainsi formées sont dites chronospecies. Evidemment, nous n'avons pas vu de tels phénomènes se dérouler sous nos yeux; il y a donc une part de reconstruction dans nos schémas, mais nous sommes devant des cas qui peuvent difficilement être interprétés autrement. $\mathrm{Si}$, dans ces conditions, une séparation géographique ou cladogenèse intervient, chacune des deux populations continuera à évoluer différemment dans sa nouvelle niche, et elles deviendront peu à peu deux espèces différentes. La cladogenèse ne peut pratiquement pas être reconnue par la paléontologie, mais elle explique admirablement l'existence d'espèces géminées que nous avons évoquée plus haut.

Ce concept d'évolution des populations a été considéré par Mayr [23] comme la plus importante révolution philosophique qu'ait connue la biologie. Cependant, les ponctualistes, notamment Gould, ont suggéré que l'anagenèse n'était que, dans de rares cas, le système de la spéciation et ils ont insisté sur le rôle que devait jouer ici l'effet bottleneck, c'est-à-dire la constitution d'une population isolée à partir d'un très petit nombre de sujets fondateurs. En outre, ils ont admis que l'espèce, après ce commencement brusque, se mettait en stase. Ce dernier fait est contredit par beaucoup d'observations. Nous évoquons plus loin l'effet bottleneck.

Le polymorphisme. Au moment de la mise en place des premières hypothèses de la théorie synthétique, on pensa que l'adaptation des espèces à leur milieu serait parfaite si elles étaient homozygotes pour tous leurs loci. Dobzhansky écrivait en plaisantant que ce système aurait abouti aux espèces idéales de Platon [20]. Très vite, on comprit que dans une population il pouvait exister, pour chaque locus, une famille possible de gènes. On appela ce phénomène le polymorphisme. En 1966, Lewontin et Hubby [24], grâce à des méthodes biochimiques, montrèrent qu'il $y$ avait souvent, chez un même sujet, moins de 10 à $20 \%$ de loci homozygotes. On admit alors que l'hétérozygotie était due à l'accumulation, au cours du temps, de petites mutations qui n'avaient pas été éliminées par la sélection. Ce phénomène donnait à chaque espèce une variabilité fabuleuse sans que les individus ne sortent pour autant du cadre de leur espèce ; cependant, il était inattendu car la sélection n'aurait pas dû laisser se maintenir ce polymorphisme. Deux explications ont été formulées pour expliquer cette difficulté : l'une supposait qu'il y avait dans le génome beaucoup de gènes neutres ou subneutres (Kimura, [25]), c'est la thèse neutraliste; l'autre suggérait 


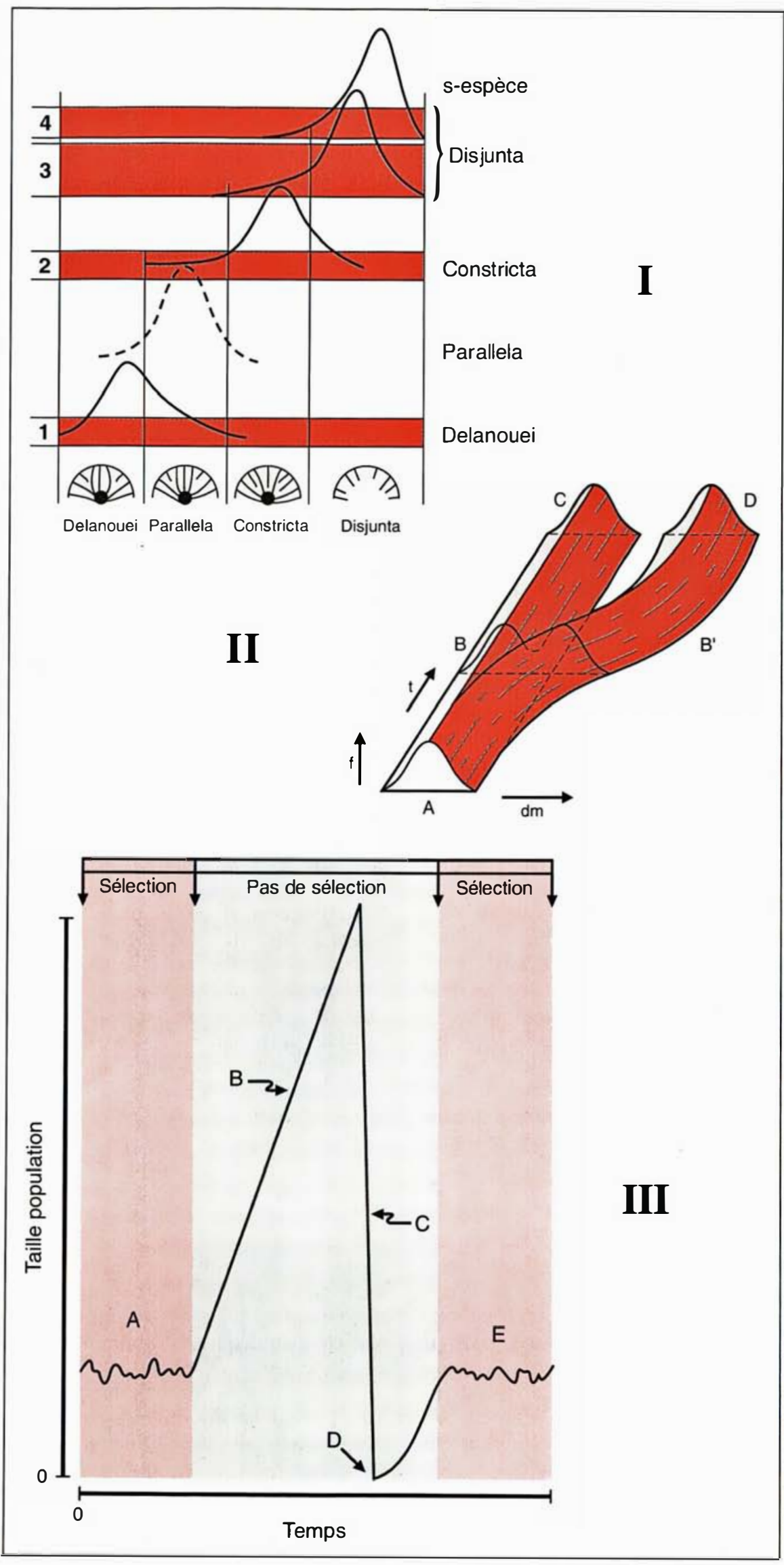

que ce phénomène était globalement un avantage car il permettait aux espèces de s'adapter facilement aux situations les plus diverses (polymorphisme équilibré). Il paraît difficile de dire aujourd'hui celui de ces mécanismes qui explique le mieux les données de Lewontin et Hubby, ou quel est le pourcentage d'explications valables que chacun apporte. Nous avons personnellement plusieurs fois changé d'avis sur cette question.

Figure 3. Il y a plusieurs modèles de spéciation, comme l'a écrit J. Génermont dans médecine/sciences [29], mais on peut considérer qu'en dehors de la polyploïdie il y a deux modèles essentiels, le système anagenèse-cladogenèse et celui de divers phénomènes correspondant à un effet bottleneck tels que l'effet fondateur, la dérive génétique, la révolution génétique. Le premier est représenté ici d'après deux schémas de Tintant, le second par un schéma de Carson. En I, on décrit l'évolution anagénétique du squelette calcaire d'une espèce de tétracoralliaire (corail) du carbonifère de la lignée Zaphrentis delanouei. Les courbes indiquent, en fonction du temps géologique (ordonnée), le pourcentage de types morphologiques correspondant à certaines dispositions des cloisons internes du corail (septa) dessinées au bas de la figure. La courbe en pointillés figure la reconstruction théorique d'une espèce intermédiaire possible dont les fossiles nont pas encore été trouvés. En II est donné un schéma théorique où l'espèce $A$ est divisée en deux populations qui divergent en donnant deux sous-espèces $B$ et $B^{\prime}$ puis deux espèces distinctes $C$ et $D$. : $d m=$ variations morphologique, $t=$ temps, $f=$ fréquence. En III on a dessiné le flushcrash de Carson avec la légende qui suit. $A$ : population sous sélection naturelle. $B$ : population en période d'expansion (flush). $C$ : population qui, à la suite d'un événement $X$, est détruite presque totalement ; les sujets qui disparaissent sont tués au hasard sans que la sélection intervienne (crash). $D$ : reste de la population survivant après le crash. E: nouvelle population se développant à la suite du flush-crash qui a entraîné une révolution génétique, et donc contribué à la naissance $d^{\prime}$ une espèce $B$ différente de A. (Parties I et II, d'après [35]), partie III, d'après [27].) 
Effet fondateur - dérive génétique - révolution génétique

- Effet bottleneck. Lorsque, d'une grande population, s'échappent quelques sujets qui fondent une colonie nouvelle, il paraît logique que dans cette petite population, le pool des gènes ne soit pas statistiquement semblable à celui de la grande dont il est issu. Cette transformation génomique pourra donner " un coup de pouce" à une spéciation ou/et en être le point de départ. Il y aura en effet en même temps " effet fondateur " et, comme le génome se réorganisera, "dérive génétique ". Cela a d'abord été l'objet de données théoriques de génétique des populations. Lamotte [26], le premier, a démontré la réalité de cette théorie en travaillant sur plusieurs milliers de petites populations d'un escargot des haies : Cepaea nemoralis Lamotte et Génermont ont défini trois niveaux d'effets fondateurs : premier ordre, action immédiate donnant une population nouvelle caractéristique (chat des Kerguelen ou de l'île Maurice) ; deuxième ordre, perturbation des génomes ; troisième ordre, perturbation des biocoenoses*.

Carson [27] et d'autres auteurs (figure 3, partie III), reprenant le concept de révolution génétique de Mayr, ont suggéré - par la théorie seulement - que, si la petite population originelle subissait ensuite un grand développement (flush) puis une réduction d'effectif considérable au hasard

* Biocoenose : groupement d'organismes vivants en équilibre instable adapté à certaines circonstances écologiques.

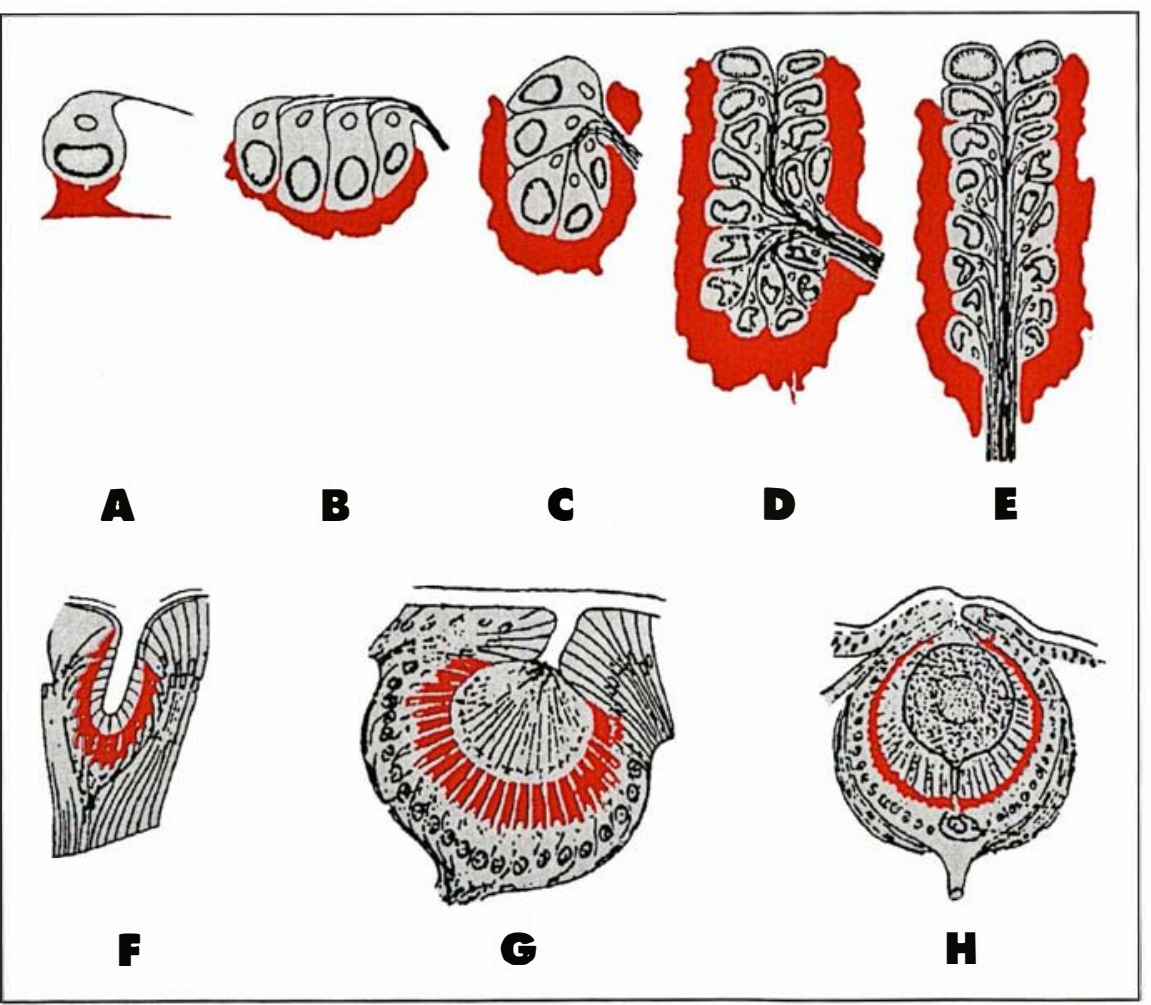

Figure 4. A partir de l'anatomie comparée d'espèces actuelles, on peut essayer de reconstituer, par analogie, des séquences phylogénétiques de I'histoire d'êtres vivants aujourd'hui disparus. Chez des métazoaires primitifs du groupe des annélides par exemple, on trouve des espèces qui présentent des ocelles ou des yeux de structure progressivement complexe. Comme le disait Teissier à ses élèves, il est évident qu'en regardant ces schémas on est amené à penser qu'une mutation triée par la sélection, plus une mutation triée par la sélection, plus... etc., peuvent construire petit à petit un organe complexe tel que l'œil. Espèce $A$ : Branchellion; espèce $B$ : Piscicola ; espèce $C$ : Nephelis; espèces $D$ et $E$ : Hirudo; espèce $F$ : Ranzania sagittarria ; espèce $G$ : Syllis aurantiaca; espèce $H$ : Phyllodoce luminosa. (D'après [30]). (crash), il pouvait se constituer très vite une espèce nouvelle, voire un genre (effet bottleneck); on a même pensé que ce système représentait le schéma principal de la spéciation. Ces phénomènes auraient alors expliqué les changements brusques proposés par les ponctualistes. Powel [28] a reconstitué le schéma de Carson. Sur huit populations étudiées, à la suite de plusieurs flush-crash, il n'a obtenu que dans trois cas un début d'isolement sexuel, c'est-à-dire un début de constitution d'espèce. On notera en outre que, dans la nature, des effets correspondant à plusieurs séries flushcrash doivent être rares. Enfin, on précisera même que l'on connaît de nombreux cas d'effets fondateurs dans la nature (Passer domesticus), même parfois des effets fondateurs successifs (Achatina fulica), et cela ne semble pas avoir provoqué plus que ce qui a été reconnu déjà : de petites modifications demeurant dans le cadre de l'espèce. Comme Génermont l'a écrit récemment dans un article de cette revue [29], il s'agit là seulement de "mécanismes accélérateurs" de la spéciation.

\section{Les organes complexes}

Souvent, les biologistes - y compris Darwin - n'arrivent pas à croire que des changements héréditaires triés au hasard par la sélection aient pu construire des organes complexes comme l'œil. Pour cet appareil, la réponse peut être résumée ainsi (figure 4).

Les zoologistes savent depuis longtemps que la transparence est une propriété qu'acquièrent parfois les tissus vivants. Il existe même un poisson téléostéen complètement transparent, que l'on reconnaît dans un aquarium parce que son squelette est noir. On sait également que l'embryogenèse se réalise par une série d'inductions en cascade, et que c'est l'ébauche de la rétine qui induit la constitution de la cornée et du cristallin. Il n'est donc pas étonnant que ces organes transparents apparaissent exactement en face de la rétine puisque c'est elle qui les induit. On notera d'ailleurs que ces phénomènes étant très généraux, on les retrouve dans beaucoup d'espèces. Il existe même un protozoaire dinoflagellé chez lequel un organite cytoplasmique joue un rôle de rétine et, en face de 
1. Delsol M Sentis Ph, Flatin J. L'Évolution biologique en vingt propositions. Essai d'analyse épistémologique de la théorie synthétique de l'évolution. Paris : Vrin - Lyon : IIEE, 1991: $850 \mathrm{p}$.

2. Lamotte $M$ (sous la direction de). Le polymorphisme dans le règne animal. $\mathrm{Mem}$ Soc Zool Fr 1974; 37 : 564 p.

3. Bock IR. Interspecific Hybridization in the Genus Drosophila, in Evolutionary Biology, vol. 18. New York : Plenum Press, 1984: 41-70.

4. Bocquet Ch, Génermont J, Lamotte $M$. Les problèmes de l'espèce dans le règne animal. Mem Soc Zool Fr 1976 ; 38 (I) : 407 ; $1977: 39$ (II) : $381 ; 1980 ; 40$ (III) : 453.

5. Jaeger JJ. Equilibres ponctués et gradualisme phylétique : un faux débat ? In : Chaline J, ed. Modalités, rythmes, mécanismes de l'évolution biologique. Dijon : CNRS 1982 ; 330 : 145-53.

6. Ouvrage collectif. La notion de vicariance : aspects biogéographique et écologiques. Actes colloque Soc Ecol Soc Biogéogr, Soc Zool Fr. Bull Ecol 1986; 17 : 215.

7. Ouvrage collectif. Les caractéristiques taxinomiques, écologiques et biogéographiques des peuplements insulaires. Bull Soc Zool Fr 1987; 112: 278.

8. Bons J. Les niches écologiques et l'isolement des espèces. In : Boms J, ed. Aspects modernes des recherches sur l'évolution. CR Colloque Montpellier, 1977. Mémoires et Travaux de l'Institut EPHE. Montpellier: EPHE, $1979 ; 10$ : 197-216.

9. Kemp TS Mammal-like Reptiles and the Origin of Mammals. London - New York: Academic Press, 1982 : 363.

10. Kemp TS. The origin of mammals : observed pattern and inferred process. In : L'Evolution dans sa réalité et ses diverses modalités. Fondation Singer-Polignac. Paris : Masson, 1988 : 65-91.

11. Denton M. Evolution. Une théorie en crise. Paris : Londreys, 1988 ; 385 p.

12. Delsol M, Flatin J. Qu'est-ce qu'une grande théorie biologique? A propos de la critique d'un ouvrage récent. Acta Biotheoretica $1992 ; 39$ : 363-73.

13. Darnell J, Lodish H, Baltimore D. La Cellule. Biologie moléculaire. Québec: Decarie - Paris : Vigot, 1988 : 1190 p.

14. Delsol M. Cause, loi, hasard en biologie. Paris : Vrin - Lyon: IIEE, 1985: 241 p.

15. Teissier G. Transformisme d'aujourd’hui. Ann Biol 1962; 1 : 359-74.

16. Petit $\mathrm{Cl}$. La concurrence larvaire et le maintien du polymorphisme, série D. $C R$ Acad Sci Paris 1966 ; 263 : 1262-5.
17. Ford EB. Génétique écologique. Paris : Gauthier-Villars, 1972： 448 p.

18. Hecht MK Natural selection in the lizard genus Aristelliger. Evolution 1952; 6 : 112-24.

19. Lerner IM. Genetic Homeostasis. Edimbourg: Oliver and Boyd, 1954: $134 \mathrm{p}$.

20. Dobzhansky Th. Génétique du processus évolutif. Paris : Flammarion, 1977: $583 \mathrm{p}$.

21. Dommergues JL. L'évolution chez les Ammonitina du Lias moyen (Carixien Domérien basal) en Europe occidentale. Doc Lab Géol Lyon 1987; 98 : 297 p

22. Gingerich PD. The stratophenetic approach to phylogeny. Reconstruction in vertebrate paleontology. In : Gracraft Eldredge, eds. Phylogenetic Analysis and Paleontology. New York: Cracraft - Eldredge, Columbia University Press, 1979 : 41-77.

23. Mayr E. Populations, espèces et évolution. Paris: Hermann, 1974: $496 \mathrm{p}$.

24. Lewontin RC, Hubby JL. A molecular approach to the study of genetic heterozygosity in natural populations. II Amoun of variation and degree of heterozygosity in natural populations of Drosophila pseudoobs cura. Genetics 1966 ; 4 : 595-609.

25. Kimura M. Théorie neutraliste de l'évolution. Paris : Flammarion, 1990: $472 \mathrm{p}$.

26. Lamotte M. Recherches sur la structure génétique des populations naturelles de Cepacea nemoralis L. Thèse, Paris. Bull Biol Fr Bels 1951; (suppl no 35) : 239 p.

27. Carson HL. The genetics of speciation at the diploid level. Am Nat $1975 ; 109$ : 83-92.

28. Powell JR. The founder-flush speciation theory. An experimental approach. Evolution $1978 ; 32$ : 465-74

29. Génermont J. Les mécanismes de la spéciation. médecine/sciences $1991 ; 6$ : I-VI

30. Salvini-Plawen L, Mayr E. On the evolution of photoreceptors and eyes. In Hecht MK, Steere WC, Wallace B, eds. Evolutionary Biology. New York, London: Plenum Press, 1977 : 207-63.

31. Goldschmidt R. The material basis of evolution. New Haven : Yale University Press, 1982 : 436 p.

32. Nardon P, Gianinazi-Pearson V, Grenier AM, Margulis L, Smith DC. Endocytobiology IV. 4th International Colloquium on endocytobiology and symbiosis. Paris : INRA, $1989: 620 \mathrm{p}$

33. Futuyma DJ. Evolutionary Biology, $2^{e}$ cd. Sunderland, Massachusetts : Sinauer Associates Inc, 1986 : $600 \mathrm{p}$.

34. Maynard Smith J. Evolutionary Genetics. Oxford : Oxford University Press, 1989 $325 \mathrm{p}$.

35. Tintant $\mathrm{H}$. Les problèmes de l'espèce dans le règne animal. Mem Soc Zool Fr 1980 ; III (40) : 359. celle-ci, il y a une zone cytoplasmique transparente assimilable à un cristallin. Lorsque l'on examine enfin la série des yeux primitifs que nous offre le monde vivant actuel, on constate que l'on peut, par analogie, imaginer la construction dans le temps, c'est-à-dire en diachronie, d'une séquence où une mutation sélectionnée, plus une mutation sélectionnée, et ainsi de suite, aboutissent à la constitution d'un œil complexe [30]. Ici, il ne faut pas oublier en outre que la rétine est une association d'unités élémentaires : c'est un système répétitif. Dans un système répétitif, lorsqu'une mutation agit sur un organite d'une cellule, elle agit sur toutes les cellules répétées.

Pour beaucoup d'organes - il est vrai en général connus seulement des spécialistes d'une science aujourd'hui oubliée, l'anatomie comparée - on pourrait montrer encore par analogie, c'est-à-dire d'après ce que l'on connaît chez les vivants actuels, que tout a pu se construire par un jeu de petites mutations sélectionnées : formation du poumon à partir de la vessie natatoire, formation du patagium (membrane de peau constituant une peudo-aile) chez les mammiferes volants, et surtout, nous l'avons déjà évoqué, passage du système circulatoire très simple des poissons à la double circulation des mammiferes supérieurs. Cela, évidemment, contredit l'ancienne thèse de Goldschmidt qui prétendait qu'il y avait des "sauts" dans l'évolution [31]. Ainsi, même pour les grands organes que nous venons de citer, la théorie synthétique admet que, hormis exceptions très rarcs toujours possibles, leur construction a été de nature graduelle.

Aucune théorie biologique ne peut jamais prétendre être achevée, hormis dans ses propositions de base telles que : toute cellule vient d'une autre cellule. La théorie de l'évolution que nous venons de résumer n'échappe pas à cette règle. Il nous semble que nous avons compris le principe du mécanisme évolutif et que nous l'avons généralisé par inférence. Toutefois, il est nécessaire que celui-ci soit enrichi de données nouvelles. En chimie, il a été possible depuis Mendeleiev de mettre à leur place tous les corps simples de la planète, mais il 
n'y en a qu'une centaine alors qu'il existe des millions d'espèces vivantes et certainement des dizaines de milliers de structures complexes. Il est probable qu'il y a aussi dans quelques cas des faits évolutifs qui pourraient relever d'une explication un peu différente de celle qu'en donne la théorie synthétique (propositions $\mathrm{XX}$ et XXI) [32]. A notre avis, ils devraient être très rares, mais l'histoirc des théorics scientifiques recèle parfois des surprises

\section{Summary}

An essay on the epistemologic analysis of Evolution

In reply to the critics and questions raised by the synthetic theory of cvolution, we have attempted to summarize here an cpistemological analysis of this theory which we have recently published [1]. This far too bricf expose wishes to emphazise both the volume and the diversity of the observations and experiments upon which the synthetic theory is based, as well as to try to describe the different processes and explanatory patterns of the biological cvolution it proposes : from the wcll-known mutation-selection system to the construction of the most complex organs or to the passage of one high taxon to another in our phylogeneses (i.c. from a great catcgory to onc another in the classification). We have especially stressed the interaction between directional and stabilizing selection.

\section{Delsol}

professeur éménte à la faculté catholique des sciences de Lyon, directeur honoraire à l'École pratique des hautes études.

Ph. Sentis

ancien sous-directeur de laboratoire au College de France.

J. Flatin

maitre de conférences à l'École pratique des hautes études.

Laboratoire de biologie générale, faculté catholique des sciences de Lyon, laboratoire d'étude du développernent post-embryonnaire des vertébrés inférieurs, Ecole pratique des hautes études, 25, rue du Plat, 6.9288 Lyon Cedex 02, France.

\section{TIRÉS A PART}

M. Delsol. 